Revista de Matemática: Teoría y Aplicaciones 1998 5(1) : 11-23

CIMPA - UCR - CCSS ISSN: 1409-2433

\title{
LA FACTORIZACIÓN DE UNA TRANSFORMADA DE FOURIER EN EL MÉTODO DE WIENER-HOPF
}

\author{
José Rosales Ortega* - Carlos Márquez Rivera ${ }^{\dagger}$ \\ Recibido: 20 Julio 1997 - Versión revisada: 25 Mayo 1998
}

\begin{abstract}
Resumen
Se factoriza la transformada de Fourier del núcleo de una ecuación integral singular, al utilizar el método de Wiener-Hopf, como el producto de dos funciones: una holomorfa en el semiplano superior y otra holomorfa en el semiplano inferior.
\end{abstract}

Palabras-clave: productos de funciones, transformada de Fourier, mtodo de WienerHopf.

\begin{abstract}
Using the Wiener-Hopf method, we factorize the Fourier Transform of the kernel of a singular integral equation as the product of two functions: one holomorphic in the upper semiplan and the other holomorphic in the lower semiplan.
\end{abstract}

Keywords: function product, Fourier transform, Wiener-Hopf method.

AMS Subject Classification: 45E10, 42A38

\section{Introducción}

El propósito de este artículo es el de "factorizar" la tranformada de Fourier del núcleo de una ecuación integral singular de la siguiente forma:

$$
\varphi(x)+\mu \int_{0}^{+\infty} \frac{\varphi(t)}{\pi(x-t)} \mathrm{d} t=f(x), \quad x \geq 0
$$

a la hora de usar el método de Wiener-Hopf, para luego utilizar tal factorización en la resolución de la ecuación (1).

\footnotetext{
*Escuela de Matemática, Instituto Tecnológico de Costa Rica, Cartago, Costa Rica.

${ }^{\dagger}$ Departamento de Matemática, Sede Regional de Occidente, Universidad de Costa Rica, San Ramn.
} 
Ha de señalarse que la solución de (1) ya ha sido encontrada para varias familias de funciones. Sin embargo al momento de resolver (1) no queda claro cómo debe factorizarce la transformada de Fourier del núcleo y además la forma de reolver la ecuación no es clara tampoco. En este artículo se demuestra la forma en que debe de hacerse esto para un caso específico de núcleo singular.

Antes de exponer nuestra idea se explicará en qué consiste el método de Wiener-Hopf para resolver ecuaciones integrales y en qué consiste el factorizar el núcleo de una ecuación integral.

\subsection{Factorización de Funciones Holomorfas}

La idea de "factorizar" una función holomorfa es una cuestión fundamental en el método de Wiener-Hopf. Es por ello que pasaremos a definir tal asunto.

Sea $\hat{K}$ la transformada de Fourier de cierta función. Supóngase que se tiene lo siguiente

$$
\hat{K}(\xi)=\hat{K}_{+}(\xi) \cdot \hat{K}_{-}(\xi), \quad \text { para } \xi \text { real }
$$

donde $\hat{K}_{+}(\xi)$ es holomorfa en $\Im(\zeta)>0$ y continua en $\Im(\zeta) \geq 0$ y $\hat{K}_{-}(\zeta)$ es holomorfa en $\Im(\zeta)<0$ y continua en $\Im(\zeta) \leq 0$ con $\zeta=\xi+i \eta$. En estas condiciones decimos que $\hat{K}$ se puede factorizar como el producto de dos funciones dotadas de un prolongamiento holomorfo.

Un ejemplo de esto lo constituye el siguiente. Sea $K(x)=1 / 2 \exp (-|x|)$, es conocido que $\hat{K}(\xi)=\left(1+\xi^{2}\right)^{-1}$. Ahora obtenemos una factorización del tipo (2) si ponemos $\hat{K}_{+}(\zeta)=(\zeta+i)^{-1}$ y $\hat{K}_{-}(\zeta)=(\zeta-i)^{-1}$.

\subsection{El Método de Wiener-Hopf}

Una ecuación integral de Wiener-Hopf de segunda especie tiene la siguiente forma

$$
\phi(x)-\int_{0}^{\infty} K(x-t) \phi(t) d t=f(x), \quad x>0
$$

En (3) la función $K$ es localmente integrable, $f$ una distribución sobre $\mathbb{R}$ y $\phi$ es la función incógnita. Además se pide que el soporte de $\phi$ esté contenido en $[0, \infty[$ y que $1-\hat{K}(\xi) \neq 0$ para $\xi$ real.

El método de Wiener-Hopf para resolver (3) consiste primero en extender el rango de integración al intervalo $]-\infty, \infty[$. Para hacer esto se considera la siguiente ecuación

$$
\operatorname{phi}(x)-\int_{-\infty}^{\infty} K(x-t) \phi(t) d t-f(x)=\psi(x), \quad-\infty<x<\infty
$$

Ahora la ecuación (3) es equivalente a (4) con la condición de que

$$
\psi(x)=0, \quad \text { siempre que } x<0
$$


Aplicando la transformada de Fourier a ambos lados de (4) y teniendo en cuenta que la trasformada de Fourier de la convolución es igual al producto de las transformadas de Fourier [5], se concluye que

$$
\hat{\psi}(\xi)=\hat{\phi}(\xi)-\hat{K}(\xi) \hat{\phi}(\xi)-\hat{f}(\xi)
$$

La ecuación (6) es equivalente a la siguiente ecuación

$$
\hat{\psi}(\xi)=\hat{\phi}(\xi)\{1-\hat{K}(\xi)\}-\hat{f}(\xi)
$$

Es aquí donde se ocupa factorizar el término $1-\hat{K}(\xi)$ en el sentido de la sección anterior. Con esto en mano la ecuación (7) se transforma en

$$
\frac{\hat{\psi}(\xi)}{\hat{K}_{+}(\xi)}-\hat{K}_{-}(\xi) \hat{\phi}(\xi)=-\frac{\hat{f}(\xi)}{\hat{K}_{+}(\xi)}
$$

Ahora si $\psi$ pertenece a una clase conveniente de funciones, su transformada de Fourier $\hat{\psi}$ se prolonga como una función de $\zeta=\xi+i \eta$, holomorfa en en semiplano $\Im(\zeta)>0$. Esto no es más que una versión del teorema de Paley-Wiener [4]. Ahora si $\psi$ es absolutamente integrable, $\hat{\psi}$ se prolonga como una función de $\zeta=\xi+i \eta$, holomorfa en $\Im(\zeta)>0$ y uniformemente en $\Im(\zeta) \geq 0$. Si buscamos la función incógnita en una conveniente clase de funciones se tendría que $\hat{\phi}$ se prolonga como una función de $\zeta=\xi+i \eta$ y holomorfa en $\Im(\zeta)<0$.

Con la explicación anterior se tiene que la resolución de la ecuación (8) es equivalente al siguiente problema: encontrar una función $\Phi$, holomorfa en los semiplanos $\Im(\zeta)>0$ y $\Im(\zeta)<0$, verificando cierta condición en el infinito y la ecuación

$$
\Phi(\xi+i \cdot 0)-\Phi(\xi-i \cdot 0)=-\frac{\hat{f}(\xi)}{\hat{K}_{+}(\xi)}, \quad \text { para } \xi \text { real } .
$$

La determinación de tal función $\Phi$ es un asunto conocido como el problema no homogéneo de Hilbert [2]. Tal función viene dada por medio de la integral de Cauchy siguiente

$$
\Phi(\zeta)=\int_{-\infty}^{\infty} \frac{-\hat{f}(t) d t}{K_{+}(t) 2 \pi i(t-\zeta)}
$$

De esto se obtiene que la solución de (8) es dada por

$$
\left\{\begin{array}{l}
\hat{\phi}(\xi)=\frac{1}{K_{-}(\xi)}[\Phi(\xi+i \cdot 0)+\text { polinomio }] \\
\hat{\psi}(\xi)=K_{+}(\xi)[\Phi(\xi-i \cdot 0)+\text { polinomio }]
\end{array}\right.
$$

Por último observe que la solución se obtiene explícitamente al tomar la transformada inversa de Fourier en las ecuaciones del párrafo anterior.

No podemos finalizar esta sección sin indicar que el método de Wiener-Hopf ha sido resuelto recientemente para una clase más amplia de núcleos: los llamados núcleos distriucionales. 


\section{Factorización de $\lambda-i \mu \operatorname{sgn} \xi$}

En esta sección se expone la parte principal del artículo. Aquí se factoriza la transformada de Fourier de cierto núcleo como el producto de dos funciones que poseen prolongaciones holomorfas.

Empezamos por encontrar la solución $\varphi$ del sistema:

$$
\begin{cases}\varphi(x)=0 & \text {, si } x<0 \\ \lambda \varphi(x)+\mu \int_{0}^{+\infty} \frac{\varphi(t)}{\pi(x-t)} \mathrm{d} t=f(x) & , \text { si } x>0\end{cases}
$$

donde $\lambda$ y $\mu$ son constantes (reales o complejas), $\mu \neq 0$ y $f$ una función conocida.

La ecuación (11) se puede considerar como una ecuación de Wiener - Hopf, con lo cual después de tomar la Transformada de Fourier, deberíamos de factorizar la siguiente función:

$$
\hat{K}(\xi)=\lambda-i \mu \operatorname{sgn} \xi
$$

El siguiente lema, cuya demostración es muy sencilla, es la clave del artículo; por medio de él se pudo obtener la factorización deseada del tipo (2).

Lema 1 Si $\lambda$ y $\mu$ son constantes (reales o complejas) diferentes de cero, entonces es válida la siguiente fórmula:

$$
\lambda-i \mu \operatorname{sgn} \xi=(\lambda-i \mu)\left(\frac{\lambda+i \mu}{\lambda-i \mu}\right)^{\frac{1-\operatorname{sgn} \xi}{2}}
$$

Definición 1 Sea $k=\sqrt{\lambda^{2}+\mu^{2}}$, donde $\lambda$ y $\mu$ son constantes y escójase $v$ tal que se cumpla que $-\frac{1}{2}<\operatorname{Re}(v)<\frac{1}{2}$ y tal que $\cos (v \pi)=\lambda / k$ y $\operatorname{sen}(v \pi)=\mu / k$.

El siguiente lema nos permite explicitar la forma que debe tener el número $v$ que fue definido anteriormente.

Lema 2 Sean $\lambda y \mu$ constantes (reales o complejas) diferentes de cero, entonces

$$
v=\frac{1}{2 \pi i} \log \left(\frac{\lambda+i \mu}{\lambda-i \mu}\right)
$$

Tenemos que

$$
\frac{\lambda}{\mu}=\frac{\exp (i v \pi)+\exp (-i v \pi)}{\exp (i v \pi)-\exp (-i v \pi)}
$$

Después de unos cálculos se obtiene lo siguiente 


$$
\begin{aligned}
(\lambda-i \mu) \exp (i v \pi) & =(\lambda+i \mu) \exp (-i v \pi) \\
\exp (i 2 v \pi) & =\frac{\lambda+i \mu}{\lambda-i \mu} \\
i 2 v \pi & =\log \left(\frac{\lambda+i \mu}{\lambda-i \mu}\right) \\
v & =\frac{1}{2 \pi i} \log \left(\frac{\lambda+i \mu}{\lambda-i \mu}\right)
\end{aligned}
$$

Lema 3 Sea $v$ definida como en el lema anterior y $\xi$ una variable real, entonces se cumple la siguiente fórmula:

$$
\begin{aligned}
& (i \xi)^{v}=\exp \left[v \log |\xi|+i v \frac{\pi}{2} \operatorname{sgn} \xi\right] \\
(i \xi)^{v}= & \exp [v \log (i \xi)] \\
= & \exp [v \log |i \xi|+i v \arg (i \xi)] \\
= & \exp [v \log |\xi|+i v(\arg (i)+\arg (\xi)+2 \pi n(i, \xi))]
\end{aligned}
$$

La demostración se dividirá en dos casos. Primero supondremos que $\xi<0$. En este caso $\arg (i)=\frac{\pi}{2} \mathrm{y} \arg (\xi)=\pi$, por otro lado [1]

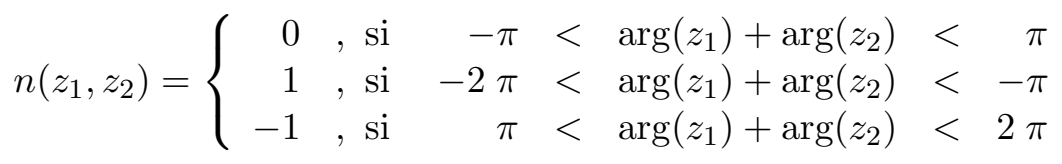

con lo cual $n(\xi, i)=-1$, combinando todo esto, obtenemos que:

$$
(i \xi)^{v}=\exp \left[v \log |\xi|-i v \frac{\pi}{2}\right]
$$

Supongamos que $\xi>0$. En este caso tenemos que $\arg (\xi)=0$ y $\arg (i)=\frac{\pi}{2}$, además $n(\xi, i)=0$, luego

$$
(i \xi)^{v}=\exp \left[v \log |\xi|+i v \frac{\pi}{2}\right]
$$

Uniendo los resultados de los casos casos anteriores, concluimos que

$$
(i \xi)^{v}=\exp \left[v \log |\xi|+i v \frac{\pi}{2} \operatorname{sgn} \xi\right] .
$$

Como caso particular del lema anterior se tiene la siguiente fórmula, la cual se obtiene al cambiar $i$ por $-i$

$$
(-i \xi)^{v}=\exp \left[v \log |\xi|-i v \frac{\pi}{2} \operatorname{sgn} \xi\right]
$$


Teorema 1 Sean $\lambda, \mu$ constantes (reales o complejas), $v$ como se definió antes y $\xi$ variable real. Entonces la función $\lambda-i \mu$ sgn $\xi$ puede factorizarse como:

$$
\lambda-i \mu \operatorname{sgn} \xi=K_{+}(\xi) K_{-}(\xi)
$$

donde $K_{+}(\xi)=(-i \xi)^{v}$ y $K_{-}(\xi)=(\lambda-i \mu) \exp [i v \pi](i \xi)^{-v}$

En primer lugar debemos observar que

$$
\left(\frac{\lambda+i \mu}{\lambda-i \mu}\right)^{\frac{1-\operatorname{sgn} \xi}{2}}=\exp [i v \pi(1-\operatorname{sgn} \xi)]
$$

Además tenemos lo siguiente

$$
\begin{aligned}
\exp [i v \pi(1-\operatorname{sgn} \xi)]= & \exp \left[i v \pi+v \log |\xi|-v \log |\xi|-i \frac{v \pi}{2} \operatorname{sgn} \xi\right. \\
& \left.+i \frac{v \pi}{2} \operatorname{sgn} \xi\right] \\
= & \exp [i v \pi] \exp \left[v \log |\xi|-i v \frac{\pi}{2} \operatorname{sgn} \xi\right] \exp [-v \log |\xi| \\
& \left.-i v \frac{\pi}{2} \operatorname{sgn} \xi\right] \\
= & \exp [i v \pi](-i \xi)^{v}(i \xi)^{v}
\end{aligned}
$$

De lo cual se sigue que

$$
\exp [i v \pi(1-\operatorname{sgn} \xi)]=(-i \xi)^{v} \exp [i v \pi](i \xi)^{-v}
$$

Ahora de las dos observaciones anteriores y del lema 1 se sigue que

$$
\lambda-i \mu \operatorname{sgn} \xi=(\lambda-i \mu) \exp [i v \pi](i \xi)^{-v}(-i \xi)^{v} .
$$

Obsérvese que $\hat{K}$ se puede factorizar como

$$
\begin{cases}K_{+}(\zeta)=(-i \zeta)^{v} & \Im(\zeta) \geq 0 \\ K_{-}(\zeta)=(\lambda-i \mu) \exp [i v \pi](i \zeta)^{-v} & \Im(\zeta) \leq 0\end{cases}
$$

Además se puede ver que

$$
K_{+}(\xi) K_{-}(\xi)= \begin{cases}\lambda-i \mu & \xi>0 \\ (\lambda-i \mu) \exp [2 v \pi i] & \xi<0\end{cases}
$$

Por lo tanto, hemos logrado factorizar la transformada de Fourier de una cierta función como el producto de otras dos funciones. 


\section{Resolución de la ecuación con núcleo singular por el mé- todo de Wiener - Hopf}

Volvamos a la ecuación (11), luego de aplicar la transformada de Fourier obtenemos que:

$$
(\lambda-i \mu \operatorname{sgn} \xi) \widehat{\varphi}(\xi)=\widehat{f}(\xi)
$$

Utilizando las mismas ideas del capítulo anterior obtenemos que por el teorema 1 la función $\lambda-i \mu \operatorname{sgn} \xi$ puede ser factorizada como el producto de dos funciones $K_{+}$y $K_{-}$ definidas por:

$$
K_{-}=(\lambda-i \mu) \exp [i v \pi](i \xi)^{-v} ; K_{+}=(-i \xi)^{v}
$$

sustituyendo estas expresiones en (12) obtenemos

$$
(\lambda-i \mu) \exp [i v \pi](i \xi)^{-v}=\Phi(\xi-i 0)
$$

donde $\Phi$ es la integral de Cauchy:

$$
\Phi(\gamma)=\int_{-\infty}^{+\infty} \frac{(-i t)^{-v} \widehat{f}(t)}{2 \pi i(t-\gamma)} \mathrm{d} t
$$

Ahora procederemos a resolver la ecuación (13), pero antes enunciaremos algunos lemas que nos facilitarán la comprensión del método.

Lema 4 Si $\widehat{\varphi}(\xi)=(i \xi)^{v} \Phi(\xi)$, entonces $\varphi(x)=\frac{d}{d x} \int_{-\infty}^{+\infty} \frac{\Phi(\xi) \exp [i x \xi]}{(i \xi)^{1-v} 2 \pi} d \xi$

Demostración. Utilizaremos la siguiente propiedad de la Transformada de Fourier, la cual es válida para la transformada inversa:

$$
\left[(i \widehat{\xi) \phi(\xi)}](x)=\frac{\mathrm{d}}{\mathrm{d} x} \widehat{\phi}(\xi)\right.
$$

Ahora, aplicando la transformada inversa a

$$
\widehat{\phi}(\xi)=(i \xi)^{v} \Phi(\xi)
$$

obtenemos

$$
\begin{aligned}
\varphi(x) & =\int_{-\infty}^{+\infty} \frac{(i \xi)^{v} \Phi(\xi) \exp [i x \xi]}{2 \pi} \mathrm{d} \xi \\
& =\int_{-\infty}^{+\infty} \frac{(i \xi) \Phi(\xi) \exp [i x \xi]}{2 \pi(i \xi)^{1-v}} \mathrm{~d} \xi
\end{aligned}
$$

Aplicando (15) a esta última integral obtenemos

$$
\varphi(x)=\frac{\mathrm{d}}{d x} \int_{-\infty}^{+\infty} \frac{\Phi(\xi)}{(i \xi)^{1-v}} \frac{\exp [i x \xi]}{2 \pi} \mathrm{d} \xi
$$


Lema 5 Si $m \in \mathbb{C}$, entonces se cumple que:

$$
(i \xi)^{-m}=\int_{0}^{+\infty} \frac{\exp [-i \mu \xi]}{\Gamma(m)} \mu^{m-1} d \mu
$$

Demostración. Considere $\Gamma(m)=\int_{0}^{+\infty} \exp [-\mu] \mu^{m-1} \mathrm{~d} \mu$

Hagamos el cambio de variable $\mu=i \xi t$, luego

$$
\begin{aligned}
\Gamma(m) & =\int_{0}^{+\infty} \exp [-i \xi t](i \xi t)^{m-1}(i \xi) \mathrm{d} t \\
& =\int_{0}^{+\infty} \exp [-i \xi t](i \xi)^{m} t^{m-1} \mathrm{~d} t
\end{aligned}
$$

Por último, obtenemos que:

$$
(i \xi)^{-m}=\int_{0}^{+\infty} \frac{\exp [-i \xi t] t^{m-1}}{\Gamma(m)} d t
$$

\section{Lema 6}

$$
\int_{-\infty}^{+\infty} \frac{\Phi(\xi) \exp [i x \xi]}{(i \xi)^{1-v} 2 \pi} d \xi=\int_{-\infty}^{x} \frac{(x-\mu)^{-v}}{\Gamma(1-v)} d \mu \int_{-\infty}^{+\infty} \frac{\Phi(\xi)}{2 \pi} \exp [i \mu \xi] d \xi
$$

Demostración. Por el lema anterior tenemos que:

$$
\begin{aligned}
(i \xi) & =\int_{-x}^{+\infty} \frac{\exp [-i(\mu+x) \xi](\mu+x)^{-v}}{\Gamma(1-v)} \mathrm{d} \mu \\
& =\int_{-\infty}^{x} \frac{\exp [i \mu \xi-i x \xi](x-\mu)^{-v}}{\Gamma(1-v)} \mathrm{d} \mu
\end{aligned}
$$

Por otro lado tenemos que:

$$
\begin{aligned}
\int_{-\infty}^{+\infty} \frac{\Phi(\xi) \exp [i x \xi]}{(i \xi)^{1-v} 2 \pi} \mathrm{d} \xi & =\int_{-\infty}^{+\infty} \frac{\Phi(\xi) \exp [i x \xi]}{2 \pi} \mathrm{d} \xi \int_{-\infty}^{x} \frac{\exp [i \mu \xi-i x \xi]}{\Gamma(1-v)(x-\mu)^{v}} \mathrm{~d} \mu \\
& =\int_{-\infty}^{x} \frac{(x-\mu)^{-v}}{\Gamma(1-v)} \mathrm{d} \mu \int_{-\infty}^{+\infty} \frac{\Phi(\xi) \exp [i \mu \xi]}{2 \pi} \mathrm{d} \xi
\end{aligned}
$$

\section{Lema 7}

$$
\int_{-\infty}^{+\infty}(-i t)^{-v} \widehat{f}(t) \frac{\exp [i \mu t]}{2 \pi} d t=\int_{\mu}^{+\infty} \frac{(t-\mu)^{v-1} f(t)}{\Gamma(v)} d t
$$

Demostración. Haremos uso de la siguiente fórmula:

$$
(-i t)^{-v}=\int_{-\infty}^{0} \frac{\exp [-i x t]|x|^{v-1}}{\Gamma(v)} \mathrm{d} x
$$


Ahora

$$
\begin{aligned}
\int_{-\infty}^{+\infty}(-i t)^{-v} \widehat{f}(t) \frac{\exp [i \mu t]}{2 \pi} \mathrm{d} t & =\int_{-\infty}^{+\infty}\left\{\int_{-\infty}^{0} \frac{e^{-i x t}|x|^{v-1}}{\Gamma(v)} \frac{\widehat{f}(t) \exp [i \mu t]}{2 \pi} \mathrm{d} x\right\} \mathrm{d} t \\
& =\int_{-\infty}^{+\infty}\left\{\int_{-\infty}^{-\mu} \frac{\exp [-i(x+\mu) t]|x+\mu|^{v-1}}{\Gamma(v)}\right. \\
& \left.\frac{\widehat{f}(t) \exp [i \mu t]}{2 \pi} \mathrm{d} x\right\} \mathrm{d} t \\
& =\int_{-\infty}^{+\infty}\left\{\int_{\mu}^{+\infty} \frac{\exp [i x t] \widehat{f}(t)}{2 \pi} \frac{|\mu-x|^{v-1}}{\Gamma(v)} \mathrm{d} x\right\} \mathrm{d} t \\
& =\int_{\mu}^{+\infty}\left\{\int_{-\infty}^{+\infty} \frac{\exp [i x t] \widehat{f}(t)}{2 \pi} \mathrm{d} t\right\} \frac{|\mu-x|^{v-1}}{\Gamma(v)} \mathrm{d} x \\
& =\int_{\mu}^{+\infty} \frac{f(x)|\mu-x|^{v-1}}{\Gamma(v)} \mathrm{d} x \\
& =\int_{\mu}^{+\infty} \frac{f(x)(x-\mu)^{v-1}}{\Gamma(v)} \mathrm{d} x
\end{aligned}
$$

Con lo cual obtenemos que:

$$
\int_{-\infty}^{+\infty}(-i t)^{-v} \widehat{f}(t) \frac{\exp [i \mu t]}{2 \pi} \mathrm{d} t=\int_{\mu}^{+\infty} \frac{(t-\mu)^{v-1} f(t)}{\Gamma(v)} \mathrm{d} t
$$

\section{Lema 8}

$$
\begin{aligned}
\int_{0}^{x}(x-\mu)^{-v} d \mu \int_{\mu}^{+\infty}(t-\mu)^{v-1} f(t) d t & =\int_{0}^{x} f(t) d t \int_{0}^{t}(x-\mu)^{-v}(t-\mu)^{v-1} d \mu \\
& +\int_{x}^{+\infty} f(t) d t \int_{0}^{x}(x-\mu)^{-v}(t-\mu)^{v-1} d \mu
\end{aligned}
$$

Demostración. Lo único que debemos hacer es un cambio en el orden de integración, pero antes observe que

$$
\int_{0}^{x}(x-\mu)^{-v} \mathrm{~d} \mu \int_{\mu}^{+\infty}(t-\mu)^{v-1} f(t) \mathrm{d} t=\int_{0}^{x} \int_{\mu}^{+\infty}(x-\mu)^{-v}(t-\mu)^{v-1} f(t) \mathrm{d} t d \mu
$$

\section{Lema 9}

$$
\int_{0}^{t}(x-\mu)^{-v}(t-\mu)^{v-1} d \mu=\int_{0}^{\frac{t}{x}} \frac{\xi^{v-1}}{1-\xi} d \xi
$$


Demostración. Hagamos la sustitución $\mu=x-\frac{x-\epsilon}{1-\epsilon}$ observe que

$$
\begin{aligned}
\int_{0}^{t}(x-\mu)^{-v}(t-\mu)^{v-1} \mathrm{~d} \mu & =\int_{0}^{t}\left(\frac{t-\mu}{x-\mu}\right)^{v-1} \frac{\mathrm{d} \mu}{x-\mu} \\
& =\int_{\frac{t}{x}}^{0} \xi^{v-1}-\frac{\mathrm{d} \xi}{1-\xi} \\
& =\int_{0}^{\frac{t}{x}} \frac{\xi^{v-1}}{1-\xi} \mathrm{d} \xi
\end{aligned}
$$

con lo cual concluimos que

$$
\int_{0}^{t}(x-\mu)^{-v}(t-\mu)^{v-1} \mathrm{~d} \mu=\int_{0}^{\frac{t}{x}} \frac{\xi^{v-1}}{1-\xi} \mathrm{d} \xi
$$

\section{Lema 10}

$$
\int_{0}^{x}(x-\mu)^{-v}(t-\mu)^{v-1} d \mu=-\int_{\frac{t}{x}}^{+\infty} \frac{\xi^{v-1}}{1-\xi} d \xi
$$

Demostración. Igual que en el lema anterior, hagamos el cambio de variable

$$
\mu=x-\frac{(x-t)}{(1-\xi)}
$$

De esto se sigue que

$$
\begin{aligned}
\int_{0}^{x}(x-\mu)^{-v}(t-\mu)^{v-1} \mathrm{~d} \mu & =\int_{0}^{x}\left(\frac{t-\mu}{x-\mu}\right)^{v-1} \frac{\mathrm{d} \mu}{x-\mu} \\
& =\int_{\frac{t}{x}}^{+\infty} \xi^{v-1}-\frac{\mathrm{d} \xi}{1-\xi} \\
& =-\int_{\frac{t}{x}}^{+\infty} \frac{\xi^{v-1}}{1-\xi} \mathrm{d} \xi
\end{aligned}
$$

\section{Lema 11}

$$
\begin{gathered}
\frac{d}{d x}\left\{\int_{x}^{+\infty} \int_{\frac{t}{x}}^{+\infty} f(t) \frac{\xi^{v-1}}{1-\xi} d \xi d t\right\}= \\
-f(x) \int_{1}^{+\infty} \frac{\xi^{v-1}}{1-\xi} d \xi+\int_{0}^{+\infty} \frac{t^{v-1} f(t)}{x^{v}(x-t)} d t
\end{gathered}
$$

\section{Demostración.}

$$
\begin{aligned}
\int_{x}^{+\infty} \int_{\frac{t}{x}}^{+\infty} f(t) \frac{\xi^{v-1}}{1-\xi} \mathrm{d} \xi \mathrm{d} t & =\int_{0}^{x} \int_{\frac{t}{x}}^{+\infty} f(t) \frac{\xi^{v-1}}{1-\xi} \mathrm{d} \xi \mathrm{d} t \\
& +\int_{0}^{+\infty} \int_{\frac{t}{x}}^{+\infty} f(t) \frac{\xi^{v-1}}{1-\xi} \mathrm{d} \xi \mathrm{d} t
\end{aligned}
$$


por otro lado tenemos que

$$
\frac{\mathrm{d}}{\mathrm{d} x} \int_{0}^{x} \int_{\frac{t}{x}}^{+\infty} f(t) \frac{\xi^{v-1}}{1-\xi} \mathrm{d} \xi \mathrm{d} t=f(x) \int_{1}^{+\infty} \frac{\xi^{v-1}}{1-\xi} \mathrm{d} \xi
$$

Observemos también que:

$$
\begin{aligned}
\frac{\mathrm{d}}{\mathrm{d} x} \int_{0}^{+\infty} \int_{\frac{t}{x}}^{+\infty} f(t) \frac{\xi^{v-1}}{1-\xi} \mathrm{d} \xi \mathrm{d} t & =\int_{0}^{+\infty} \frac{\mathrm{d}}{\mathrm{d} x} \int_{\frac{t}{x}}^{+\infty} f(t) \frac{\xi^{v-1}}{1-\xi} \mathrm{d} \xi \mathrm{d} t \\
& =-\int_{0}^{+\infty} f(t) \frac{\left(\frac{t}{x}\right)^{v-1}}{1-\left(\frac{t}{x}\right)}-\frac{t}{x^{2}} \mathrm{~d} t \\
& =\int_{0}^{+\infty} f(t) \frac{t^{v-1}}{x^{v-1}} \frac{x}{x-t} \frac{t}{x^{2}} \mathrm{~d} t \\
& =\int_{0}^{+\infty} \frac{f(t) t^{v-1}}{x^{v} x-t} \mathrm{~d} t
\end{aligned}
$$

\section{Lema 12}

$$
\begin{array}{r}
\frac{d}{d x}\left\{\int_{0}^{x} \int_{0}^{\frac{t}{x}} f(t) \frac{\xi^{v-1}}{1-\xi} d \xi d t-\int_{x}^{+\infty} \int_{\frac{t}{x}}^{+\infty} f(t) \frac{\xi^{v-1}}{1-\xi} d \xi d t\right\}= \\
\pi \cot (v \pi) f(x)-x^{-v} \int_{0}^{+\infty} \frac{t^{v-1} f(t)}{x-t} d t
\end{array}
$$

\section{Demostración.}

$$
\begin{array}{r}
\frac{\mathrm{d}}{\mathrm{d} x}\left\{\int_{0}^{x} \int_{0}^{\frac{t}{x}} f(t) \frac{\xi^{v-1}}{1-\xi} \mathrm{d} \xi \mathrm{d} t-\int_{x}^{+\infty} \int_{\frac{t}{x}}^{+\infty} f(t) \frac{\xi^{v-1}}{1-\xi} \mathrm{d} \xi \mathrm{d} t\right\}= \\
f(x) \int_{0}^{1} \frac{\xi^{v-1}}{1-\xi} \mathrm{d} \xi+f(x) \int_{1}^{+\infty} \frac{\xi^{v-1}}{1-\xi} \mathrm{d} \xi-\int_{0}^{+\infty} \frac{t^{v-1} f(t)}{x^{v}(x-t)} \mathrm{d} t= \\
f(x) \int_{0}^{+\infty} \frac{\xi^{v-1}}{1-\xi} \mathrm{d} \xi-x^{-v} \int_{0}^{+\infty} \frac{t^{v-1} f(t)}{x-t} \mathrm{~d} t= \\
f(x) \pi \cot (v \pi)-x^{-v} \int_{0}^{+\infty} \frac{t^{v-1} f(t)}{x-t} \mathrm{~d} t
\end{array}
$$

Regresemos ahora a la ecuación (12)

$$
(\lambda-i \mu \operatorname{sgn} \xi) \widehat{\varphi}(\xi)=\widehat{f}(\xi)
$$

Utilizando el teorema 1 y las ideas del método de Wiener - Hopf, expuestas en la sección anterior se concluye que

$$
(\lambda-i \mu) \exp [i v \pi](i \xi)^{-v} \widehat{\varphi}(\xi)=\Phi(\xi)
$$


Aplicando los lemas 4 y 5 a la ecuación (16), obtenemos

$$
\begin{aligned}
(\lambda-i \mu) \exp [i v \pi](i \xi)^{-v} \widehat{\varphi}(\xi)= & \frac{\mathrm{d}}{\mathrm{d} x} \int_{-\infty}^{+\infty} \frac{\Phi(\xi) \exp [i x \xi]}{(i \xi)^{1-v} 2 \pi} \mathrm{d} \xi \\
= & \frac{\mathrm{d}}{\mathrm{d} x} \int_{-\infty}^{x} \frac{(x-\mu)^{-v}}{\Gamma(1-v)} \mathrm{d} \mu \\
& \int_{-\infty}^{+\infty} \frac{\Phi(\xi) \exp [i \mu \xi]}{2 \pi} \mathrm{d} \xi
\end{aligned}
$$

Es conocido que [4]

$$
\int_{-\infty}^{+\infty} \Phi(\xi) \frac{\exp [i \mu \xi]}{2 \pi} \mathrm{d} \xi=\left\{\begin{array}{ll}
\int_{-\infty}^{+\infty}(-i t)^{-v} \widehat{f}(t) \frac{\exp [i \mu t]}{2 \pi} \mathrm{d} t & , \text { si } \mu>0 \\
0 & , \text { si } \mu<0
\end{array} .\right.
$$

Utilizando el lema 5, obtenemos

$$
\int_{-\infty}^{+\infty}(-i t)^{-v} \widehat{f}(t) \frac{\exp [i \mu t]}{2 \pi} \mathrm{d} t=\int_{\mu}^{+\infty} \frac{(t-\mu)^{v-1} f(t)}{\Gamma(v)} \mathrm{d} t
$$

De esto se sigue que (17) se reescribe como

$$
(\lambda-i \mu) \exp [i \pi v] \varphi(x)=\frac{\mathrm{d}}{\mathrm{d} x} \int_{-\infty}^{x} \frac{(x-\mu)^{-v}}{\Gamma(1-v)} \mathrm{d} \mu \int_{\mu}^{+\infty} \frac{(t-\mu)^{v-1} f(t)}{\Gamma(v)} \mathrm{d} t
$$

Utilizando el hecho de que $\Gamma(1-v) \Gamma(v)=\frac{\pi}{\operatorname{sen}(v \pi)}$, obtenemos

$$
(\lambda-i \mu) \exp [i \pi v] \varphi(x)=\frac{\mathrm{d}}{\mathrm{d} x} \int_{-\infty}^{x}(x-\mu)^{-v} \mathrm{~d} \mu \int_{\mu}^{+\infty}(t-\mu)^{v-1} f(t) \mathrm{d} t
$$

Usando los lemas 8 y 11 en $((19))$, se llega a que

$$
\begin{aligned}
\frac{\pi}{\mu}\left(\lambda^{2}+\mu^{2}\right) \varphi(x)= & \frac{\mathrm{d}}{\mathrm{d} x} \int_{0}^{x}(x-\mu)^{-v} \mathrm{~d} \mu \int_{\mu}^{+\infty}(t-\mu)^{v-1} f(t) \mathrm{d} t, \text { si } x>0 \\
= & \frac{\mathrm{d}}{\mathrm{d} x}\left\{\int_{0}^{x} f(t) \mathrm{d} t \int_{0}^{t}(x-\mu)^{-v}(t-\mu)^{v-1} \mathrm{~d} \mu\right. \\
& \left.+\int_{x}^{+\infty} f(t) \mathrm{d} t \int_{0}^{x}(x-\mu)^{-v}(t-\mu)^{v-1} \mathrm{~d} \mu\right\} \\
= & \frac{\mathrm{d}}{\mathrm{d} x}\left\{\int_{0}^{x} \int_{0}^{t} f(t)(x-\mu)^{-v}(t-\mu)^{v-1} \mathrm{~d} \mu \mathrm{d} t\right. \\
& \left.+\int_{x}^{+\infty} \int_{0}^{x} f(t)(x-\mu)^{-v}(t-\mu)^{v-1} \mathrm{~d} \mu \mathrm{d} t\right\} \\
= & \frac{\mathrm{d}}{\mathrm{d} x}\left\{\int_{0}^{x} \int_{0}^{\frac{t}{x}} f(t) \frac{\xi^{v-1}}{1-\xi} \mathrm{d} \xi \mathrm{d} t-\int_{x}^{+\infty} \int_{\frac{t}{x}}^{+\infty} f(t) \frac{\xi^{v-1}}{1-\xi} \mathrm{d} \xi \mathrm{d} t\right\} \\
= & f(x) \pi \cot (v \pi)-x^{-v} \int_{0}^{+\infty} \frac{t^{v-1} f(t)}{x-t} \mathrm{~d} t
\end{aligned}
$$


Esta última igualdad se obtuvo usando los lemas 9, 10 y 12.

En resumen, hemos obtenido que:

$$
\begin{gathered}
\frac{\pi}{\mu}\left(\lambda^{2}+\mu^{2}\right) \varphi(x)=f(x) \pi \cot (v \pi)-x^{-v} \int_{0}^{+\infty} \frac{t^{v-1} f(t)}{x-t} \mathrm{~d} t \\
\left(\lambda^{2}+\mu^{2}\right) \varphi(x)=\lambda f(x)-\mu x^{-v} \int_{0}^{+\infty} \frac{t^{v-1} f(t)}{x-t} \mathrm{~d} t
\end{gathered}
$$

Esta fórmula coincide con la fórmula encontrada en [4] cuando resuelve la ecuación (12) por otros métodos.

\section{Bibliografía}

[1] Apostol, T. (1982) Anlisis Matemtico. Editorial Revert, Barcelona.

[2] Gjov, F.D. (1980) Problemas de Contorno. Editorial Mir, Mosc.

[3] Krein, M.G. (1963) "Integral equation on a half-line with kernels depending upon the difference of the arguments", American Math. Assoc. Trans. 22: 163-288.

[4] Talenti, G. (1973) "Sulle equazioni integrali di Wiener-Hopf", Boll. Un. Mat. Ital. 7(4): $18-118$.

[5] Rudin, W. (1974) Real and Complex Analysis. McGraw-Hill, New York. 